\begin{tabular}{|c|l|}
\hline Title & Controlled formation of metallic nanoball ls during plasma electrolysis \\
\hline Author(s) & Toriyabe, Yu; W atanabe, Seiichi; Y atsu, Shigeo; Shibay ama, Tamaki; Mizuno, Tadahiko \\
\hline Citation & $\begin{array}{l}\text { A pplied Physics Letters, 91(4), 041501 } \\
\text { https://doi.org/10.1063/2760042 }\end{array}$ \\
\hline Issue Date & 2007-07-23 \\
\hline Doc URL & http://hdl.handle.net/2115/30122 \\
\hline Rights & Copyright $\odot 2007$ A merican Institute of Physics \\
\hline Type & article \\
\hline File Information & APL91-04.pdf \\
\hline
\end{tabular}

Instructions for use 


\title{
Controlled formation of metallic nanoballs during plasma electrolysis
}

\author{
Yu Toriyabe ${ }^{a)}$ \\ Graduate School of Engineering, Hokkaido University, Sapporo 060-8628, Japan \\ Seiichi Watanabe, ${ }^{\text {b) }}$ Shigeo Yatsu, and Tamaki Shibayama \\ Center for Advanced Research of Energy Conversion Materials, Hokkaido University, N13, W8, Kitaku, \\ Sapporo 060-8628, Japan \\ Tadahiko Mizuno \\ Graduate School of Engineering, Hokkaido University, Sapporo 060-8628, Japan
}

(Received 11 May 2007; accepted 26 June 2007; published online 26 July 2007)

\begin{abstract}
Formation of spherical nanoparticles (hereafter "nanoballs") in a gas/liquid mixed dual phase system during plasma electrolysis is reported. A gas/vapor sheath is formed at the electrode/ electrolyte interface when the applied voltage is high enough to induce discharge plasma. Through this nonequilibrium process, the authors have produced $\mathrm{Ni}, \mathrm{Ti}, \mathrm{Ag}$, and $\mathrm{Au}$ metallic nanoballs from the cathode mother materials with a certain size controllability. The electrode surface is partially melted by the local current concentration induced by electrothermal instability followed by an immediate cooldown, yielding nanoballs without contamination from electrolyte. () 2007 American Institute of Physics. [DOI: 10.1063/1.2760042]
\end{abstract}

Although material/chemical processes are usually conducted in the gas or liquid monophase, we may expect advantages in the gas/liquid mixed dual phase, such as in liquid discharge experiments. Because of the seminal work on discharge electrolysis, ${ }^{1-4}$ it has been widely recognized as a potential method for chemical and material engineering. A salient feature of this process is the non-Faradaic reactions, ${ }^{1-3,5,6}$ ranging from polymerization ${ }^{7,8}$ to the formation of amino acids ${ }^{9,10}$ and degradation of harmful substances. ${ }^{11,12}$ The amount of product is generated over Faraday's law, which strictly predicts that the amount is exactly equal to the amount of current, mainly through anodic plasma electrolysis. Theoretical models to explain nonFaradaic reactions have been proposed. ${ }^{1,3,6,8}$ Recently, nonFaradaic hydrogen evolution has been confirmed as the direct pyrolytic decomposition of water molecules to apply it for the hydrogen economy. ${ }^{13}$ From the viewpoint of material engineering, an oxide coating method has been developed under the anodic plasma electrolysis condition as a more inexpensive, faster, and easier method as compared with previous procedures. ${ }^{14}$ Furthermore, the method has a potential to enrich a certain isotope. ${ }^{15,16}$

In this letter, we report metallic nanoballs formation at the electrode surface by plasma electrolysis with a relatively low cell voltage of less than $160 \mathrm{~V}$. The experimental setup simply consisted of a glass cell to induce glow discharge in an electrolytic solution under atmosphere pressure without a vacuum chamber or pumps. The discharge electrode was not the anode, but cathode, which could discharge at a lower cell voltage $(\sim 140 \mathrm{~V})$ than the anode. ${ }^{6,14}$ Several metallic wires, viz. $\mathrm{Ni}, \mathrm{Ti}, \mathrm{Ag}$, and $\mathrm{Au}$, were electrolyzed in a $0.1 \mathrm{~mol} / \mathrm{dm}^{3}$ $\mathrm{K}_{2} \mathrm{CO}_{3}$ solution. The counterelectrode was a Pt mesh whose surface area was much larger than that of the working electrode in order to concentrate the voltage drop at the cathode/ electrolyte interface.

\footnotetext{
${ }^{\text {a) }}$ Present address: Laboratory of Nuclear Science, Tohoku University, Sendai 982-0826, Japan.

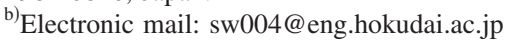

It is shown in Fig. 1(a) that glow discharge with light emission occurred only near the cathode surface area where excited atoms drop to a stable level by emitting unique energy photons as visible light. Figure 1(b) then shows the current-voltage relationship during electrolysis. We defined regions 4 and 5 as the plasma regions: (1) conventional region, (2) breakdown point, (3) transitional region, (4) partial plasma region, and (5) full plasma region. Region 1 is the standard electrolysis region with normal electrode reactions where current increases according to Ohmic law. Since the IR loss was concentrated at the cathode/electrolyte interface, the temperature near the cathode increased rapidly and generated a vapor sheath with hydrogen when the cathode temperature exceeded the boiling point of the electrolyte (regions 2 and 3). The current cannot increase any more thereafter and thus decreases because the electrode and elec-

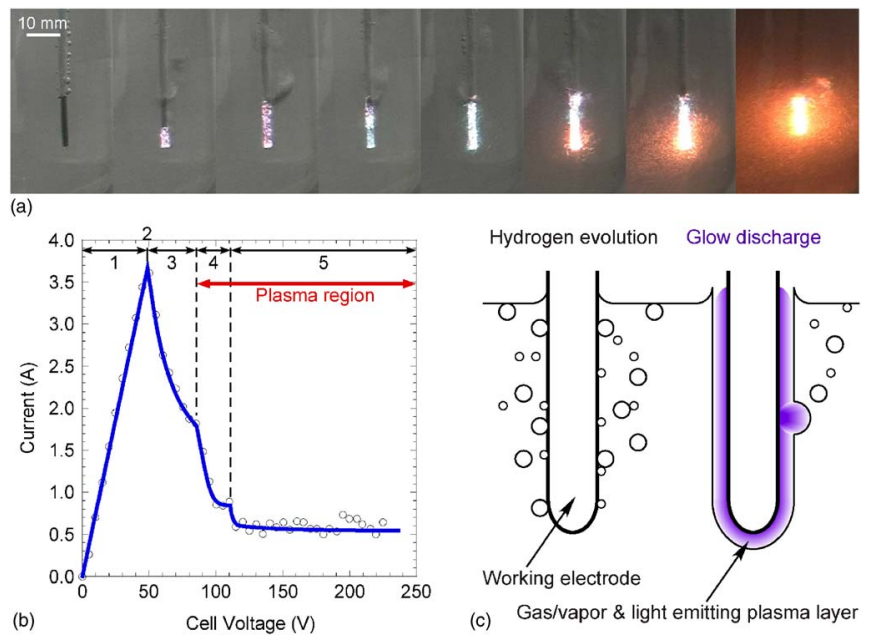

FIG. 1. (Color online) Typical current-voltage relation curve and schematic diagram before and during plasma electrolysis. (a) Pictures of Ni electrode with a diameter of $1.5 \mathrm{~mm}$ during plasma electrolysis. (b) Current-voltage relation during plasma electrolysis with $\mathrm{Ni}$ electrode. (c) A schematic diagram of plasma electrolysis; a thin gas/vapor layer is formed at the metal/ solution interface, where a high applied voltage induces the glow discharge. 

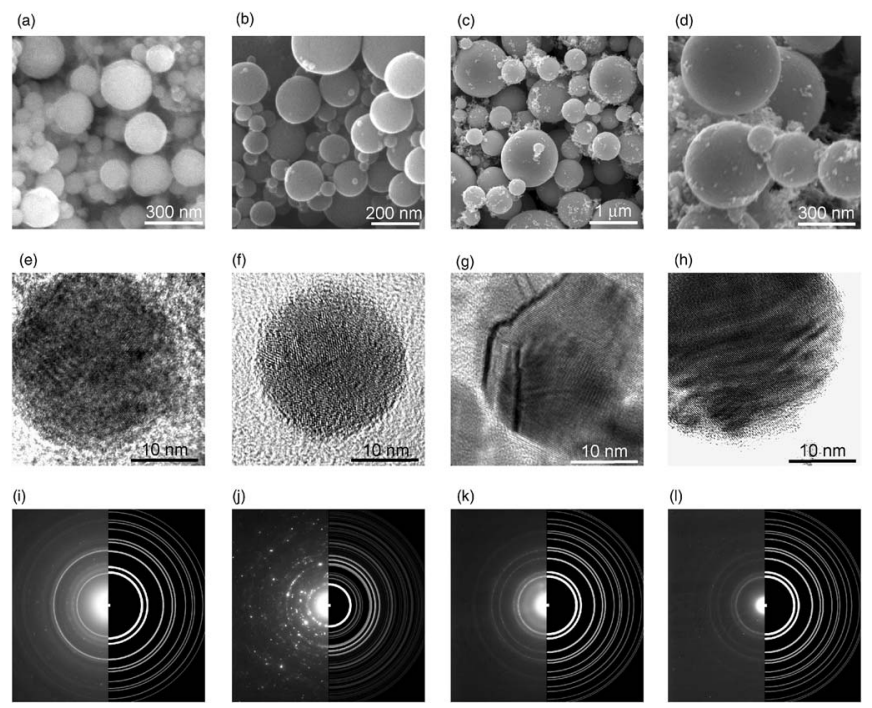

FIG. 2. Typical micrographs of Ni, Ti, Ag, and Au nanoballs observed by SEM and TEM with electron diffraction patterns. [(a)-(d)] SEM images of (a) $\mathrm{Ni}$, (b) $\mathrm{Ti}$, (c) Ag, and (d) Au electrode surfaces. [(e)-(h)] TEM images of (e) $\mathrm{Ni}$, (f) Ti, (g) Ag, and (h) Au spherical nanoparticles. [(i)-(1)] Electron diffraction patterns of (i) Ni, (j) Ti, (k) Ag, and (l) Au. The right side of each diffraction pattern is a numerical simulation assuming powder samples.

trolyte do not touch each other through the unstable region 4. If the cell voltage is sufficiently high, a glow discharge with intense light emission starts in the gas film and the light emission area expands to the whole area of the electrode in region 5 .

This plasma electrolytic process is schematically illustrated in Fig. 1(c) to show a thin gas/vapor layer formed at the metal/solution interface. The generation of the vapor/gas sheath is a key factor because the ignition condition is determined by the thickness of a sheath and the gas pressure, namely, Paschen's law. ${ }^{17,18}$ The electrochemical conditions, i.e., the type and surface area of the electrode, and the type and concentration of the electrolyte, do not influence the critical voltage over the full plasma region, which is almost $120-160 \mathrm{~V}$ in each case, while this voltage is theoretically predicted as $80-200 \mathrm{~V} .{ }^{14}$ Since the current keeps almost constant at a low level during the plasma electrolysis process, this is classified as a glow discharge. Electrons in the plasma layer are accelerated under a high electric field and impact a neutral atom to dissolve and excite it. The electronically excited atoms then, drop down immediately to a stable energy level by emitting photons. Since the excitation energies are characteristic for atom species, the color of the emitted light depends on the sort of electrodes and electrolytes. ${ }^{19,20}$ However, the Pt anode does not change from the normal anode reaction.

After plasma electrolysis, the electrode surfaces were observed by a scanning electron microscope (SEM) (JEOL JSM-6500F). Nanoballs with diameters ranging from $10 \mathrm{~nm}$ to $1 \mu \mathrm{m}$ were formed where the discharge covers, as shown in Figs. 2(a)-2(d), (a) Ni, (b) Ti, (c) Ag, and (d) Au electrode surfaces. These particles are distributed evenly over the entire cathode surface without a deviation in number and diameter, as are the discharge characteristics. Some nanoballs were scattered in the solution during the discharge process and precipitated at the bottom of the cell. After rinsed with purified water, they were collected and deposited on a $\mathrm{Cu}$ microgrid with a diameter of $3 \mathrm{~mm}$ for transmission electron microscopy (TEM) (JEOL JEM-2010F) observation. TEM images and electron diffraction patterns of corresponding metals are shown in Figs. 2(e)-2(h) and Figs. 2(i)-2(l), respectively.

Some particles' surfaces were oxidized through the nanoball formation process due to the high temperature environment or the after treatment exposure to the air, especially in the case of Ti particles. Ti particles have $\mathrm{TiO}_{2}$ surface layers, whose composition is determined by energy dispersive $\mathrm{x}$-ray analysis. The electron diffraction patterns are shown on the left side of Figs. 2(i)-2(1), while the corresponding numerical simulation ones with an assumption of powder samples were displayed on the right. Experimental electron diffraction patterns of $\mathrm{Ni}, \mathrm{Ag}$, and $\mathrm{Au}$ agree with those of the monometals, while that of $\mathrm{TiO}_{2}$ corresponds to rutile structure. The $\mathrm{x}$-ray diffraction supports the previous results.

Almost all particles greater than $10 \mathrm{~nm}$ in diameter are perfectly spherical, which minimizes the total surface area. For the sake of universality, we can apply the above process to four different metal electrodes, namely, $\mathrm{Ni}, \mathrm{Ti}, \mathrm{Ag}$, and $\mathrm{Au}$, as shown in Figs. 2(a)-2(h). The process used was exactly the same in all cases. The size distribution obtained was different in each case. At present, we believe that the electroand thermal conductivity, chemical potential, and surface energy of the electrode material play an important role in determining the particle formation processes and diameter distribution.
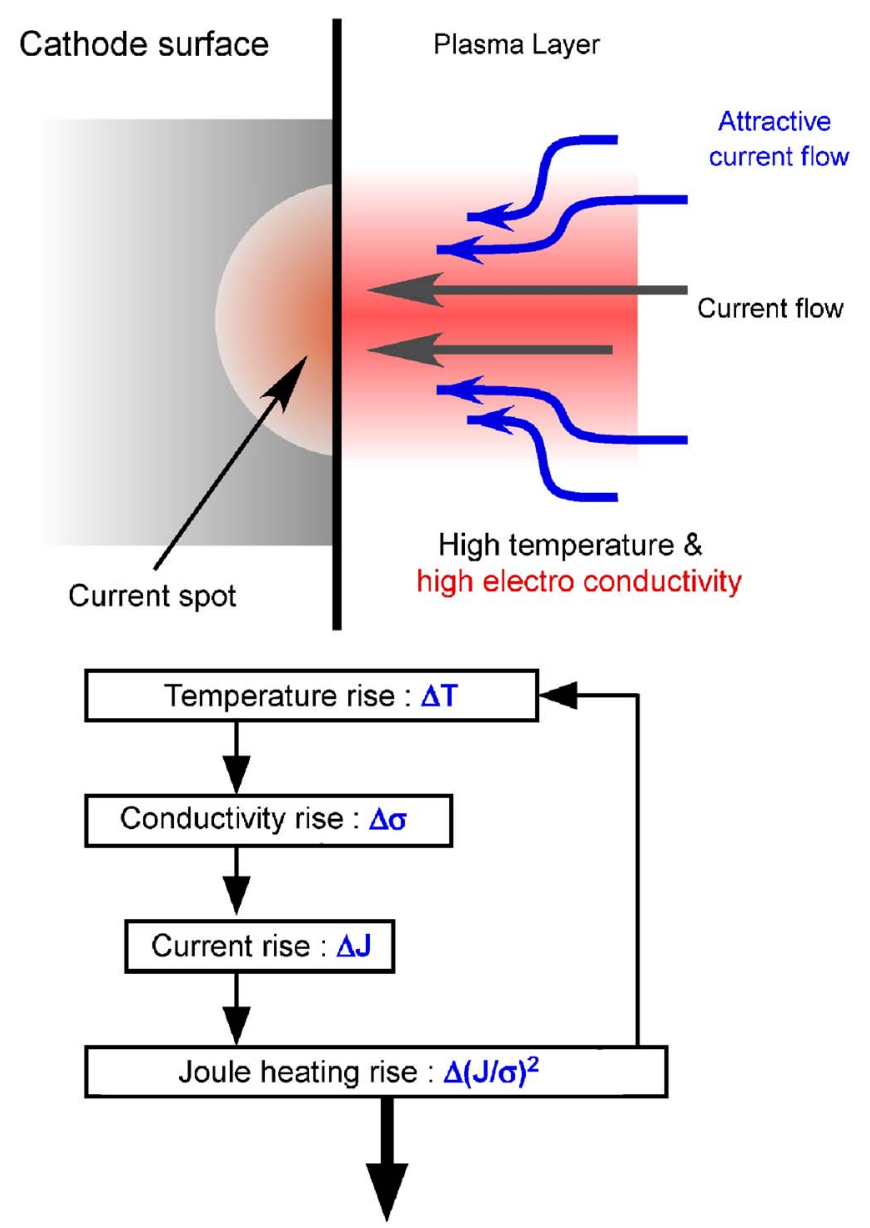

Electrode melting \& nano particle formation

FIG. 3. (Color online) Electrothermal instability scheme to explain the nanoball formation process.

o AIP license or copyright, see http://apl.aip.org/apl/copyright.jsp 


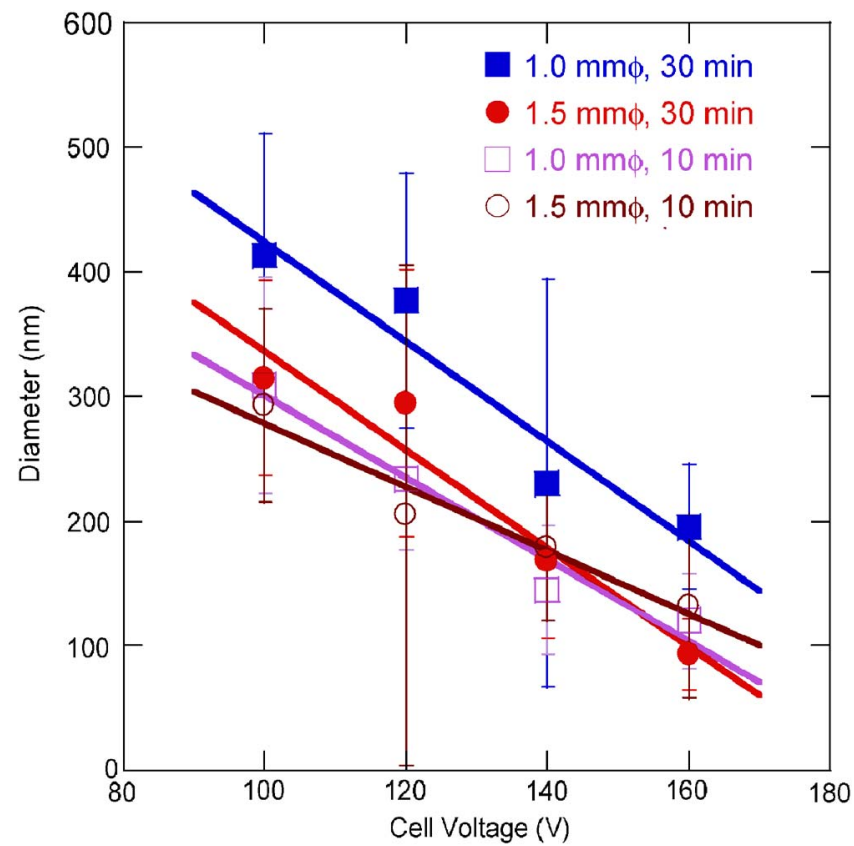

FIG. 4. (Color online) Cell voltage dependence on the diameter for $\mathrm{Ni}$ nanoballs. The mean diameter is statistically analyzed by SEM images of the electrode surface for four different voltage conditions in the case of $\mathrm{Ni}$ electrodes. The error bars represent the standard deviation.

The TEM observation reveals lattice defects of dislocations and grain boundaries or twins inside the even smaller particles. These results indicate that the electrode surface was locally heated and momentarily exceeded the melting point, and the molten metal yielded to a spherical shape due to surface tension. The metal immediately cools down to form fine spherical particles. Thus, the particle size and shape are determined by the amount of inputted heat and duration at the plasma electrolytic conditions. Since the cause of the temperature increase is Joule heating in this case, current concentration at a certain spot results in a rather large heating locally. This concentration can be explained by electrothermal instability, ${ }^{21-25}$ well recognized in plasma physics, schematically illustrated in Fig. 3. If the current at a certain spot is larger than in the surrounding area, the amount of Joule heating increases. This means that the plasma near the current concentration spot is heated extra by the added Joule heating and the temperature of the plasma state rises above the steady-state value. Since the plasma electroconductivity is an exponential function of the temperature, the conductivity increases rapidly with temperature. Thus, the current becomes larger and the cycle is repeated. Eventually, the electrode melts and yields metallic nanoballs.

Figure 4 plots the mean diameter variation of $\mathrm{Ni}$ nanoballs measured from SEM micrographs under the four cell voltage conditions. The mean value of the diameter of generated nanoballs has a tendency to decrease with increasing applied voltage, not only in the case of $\mathrm{Ni}$, but also for the other elements. The current concentration caused by electrothermal instability continues until thermal diffusion becomes equal to the Joule heating, which was explained by the previous electrothermal instability arguments. It is there- fore reasonable that the increase in current concentration can promote the process when the surrounding temperature is relatively low, i.e., under a low cell voltage. Consequently, the nanoballs generated under lower cell voltages have larger mean diameters. This also indicates that we can obtain nanoballs in the mean diameter of sub-100-nm size, if we increase the cell voltage up to several hundreds of volts.

In the present study, we report the possibility to control nanostructure through plasma electrolysis, which can be achieved in a gas/liquid dual phase with relative ease. Fine metallic nanoballs of $\mathrm{Ni}, \mathrm{Ti}, \mathrm{Ag}$, and $\mathrm{Au}$ have been formed by this nonequilibrium plasma discharge method with a certain size controllability. While previous conventional methods apply arc discharge in a vacuum chamber, including a recent one of irradiation with electrons, ${ }^{26}$ our glow discharge method in liquid can be achieved extremely easily, quickly, and inexpensively as the demand for nanoballs for industrial needs recently. Plasma electrolysis has a number of features that enable it to control such nanoball properties. Complex composition or multilayered balls ${ }^{27}$ could be obtained if we can utilize the salient feature of the gas/liquid dual phase.

One of the authors (Y.T.) wishes to thank Tadayoshi Ohmori and Yoshiaki Aoki for their great support on plasma electrolysis. The authors also thank Takanori Suda for his help in SEM observation.

${ }^{1}$ A. Hickling and M. D. Ingram, J. Electroanal. Chem. 8, 65 (1964).

${ }^{2}$ A. Hickling and M. D. Ingram, Trans. Faraday Soc. 60, 783 (1964).

${ }^{3}$ A. Hickling, Modern Aspects of Electrochemistry (Plenum, New York, 1971), Vol. 6, p. 329.

${ }^{4}$ H. H. Kellogg, J. Electrochem. Soc. 97, 133 (1950).

${ }^{5}$ S. K. Sengupta and O. P. Singh, J. Electroanal. Chem. 301, 189 (1991).

${ }^{6}$ S. K. Sengupta, R. Singh, and A. K. Srivastava, J. Electrochem. Soc. 145, 2209 (1998).

${ }^{7}$ A. R. Denaro and K. O. Hough, Electrochim. Acta 18, 863 (1973).

${ }^{8}$ A. R. Denaro, Electrochim. Acta 20, 669 (1975).

${ }^{9}$ K. Harada and T. Iwasaki, Nature (London) 250, 426 (1974).

${ }^{10}$ K. Harada and S. Suzuki, Nature (London) 266, 275 (1977).

${ }^{11}$ Q. Lu, J. Yu, and J. Gao, J. Hazard. Mater. B136, 526 (2006).

${ }^{12}$ J. Gao, J. Yu, Y. Li, X. He, L. Bo, L. Pu, W. Yang, Q. Lu, and Z. Yang, J. Hazard. Mater. B137, 431 (2006).

${ }^{13}$ T. Mizuno, T. Akimoto, K. Azumi, T. Ohmori, Y. Aoki, and A. Takahashi, Jpn. J. Appl. Phys., Part 1 44, 396 (2005).

${ }^{14}$ A. L. Yerokhin, X. Nie, A. Leyland, A. Matthews, and S. J. Dowey, Surf. Coat. Technol. 122, 73 (1999).

${ }^{15}$ T. Ohmori, H. Yamada, S. Narita, T. Mizuno, and Y. Aoki, J. Appl. Electrochem. 33, 643 (2003).

${ }^{16} \mathrm{~T}$. Ohmori, New Topics in Electrochemistry Research (Nova, New York, 2006), p. 47.

${ }^{17}$ F. Paschen, Ann. Phys. 273, 69 (1889)

${ }^{18}$ J. E. Almy, Phys. Rev. 24, 50 (1907).

${ }^{19}$ K. Doblhofer and H. Gerischer, J. Electroanal. Chem. 65, 101 (1975).

${ }^{20}$ K. Azumi, T. Mizuno, T. Akimoto, and T. Ohmori, J. Electrochem. Soc. 146, 3374 (1999).

${ }^{21}$ J. L. Kerrebrock, AIAA J. 2, 1072 (1964).

${ }^{22}$ A. H. Nelson, AIAA J. 8, 1753 (1970).

${ }^{23}$ T. Kon, N. Kayukawa, Y. Ozawa, and Y. Aoki, Proceedings of the 16th Symposium on the Engineering Aspects of MHD, Pittsburgh, PA (Energy Research and Development Administration, 1977), Paper No. VI.1.1.

${ }^{24}$ K. Okazaki, Y. Mori, K. Hijikata, and K. Ohtake, AIAA J. 16, 334 (1978).

${ }^{25}$ R. Rosa, L. Farrar, and D. Trundnowski, J. Propul. Power 4, 466 (1988).

${ }^{26}$ T. Kameyama and S.-I. Tanaka, J. Ceram. Soc. Jpn. 112, S930 (2004).

${ }^{27}$ B. S. Xu and S.-I. Tanaka, Acta Mater. 46, 5249 (1998). 